\title{
Efeito do gesso nas propriedades químicas do solo sob dois sistemas de manejo
}

\section{Gypsum effects on soil chemical properties under two management systems}

\author{
Fábio Régis de Souza ${ }^{1 *}$; Edgard Jardim Rosa Junior ${ }^{2}$; \\ Carlos Ricardo Fietz ${ }^{3}$; Anderson Cristian Bergamin ${ }^{4}$; \\ Yara Brito Chaim Jardim Rosa ${ }^{2}$; Walmes Marques Zeviani ${ }^{5}$
}

\section{Resumo}

A combinação de gessagem e manejo do solo podem influenciar as propriedades químicas do solo. Neste contexto, o objetivo deste trabalho foi avaliar as propriedades químicas do solo submetido a dois manejos do solo e ao efeito residual de gesso, após 55 meses de implantação dos tratamentos. Desenvolvido em uma área experimental da FCA/UFGD de Dourados, MS, o experimento foi disposto no delineamento de blocos casualizados com os fatores arranjados em parcela subsubdividida. A parcelas, sub-parcelas e sub-subparcelas receberam os níveis dos fatores sistema plantio direto e convencional, gesso e profundidade $0-0,05,0,05-0,10,0,10-0,15,0,15-0,20$ e $0,20-0,30 \mathrm{~m}$, respectivamente, com quatro repetições. Não houve efeito residual de gesso para cálcio. O plantio direto com efeito residual de gesso aumentou o teor de potássio, enxofre e reduziu a acidez potencial. O plantio convencional com efeito residual de gesso aumentou a acidez potencial do solo. O plantio direto promoveu aumento de cálcio (em 0-0,05 e 0,05-0,10 m), aumento do teor de matéria orgânica (em 0-0,05 m), acúmulo de enxofre (em 0,05-0,10 e 0,10-0,15 m), aumento da soma de base e saturação por base (em 0,05-0,10 m,) e redução de magnésio (em 0,10-0,15 m), fósforo (em 0,05-0,10 e 0,10-0,15 m), acidez potencial (em 0,05-0,10 m) e CTC (em 0,10-0,15 m). O efeito residual de gesso acumulou mais enxofre e saturação por base na profundidade de $0,20-0,30 \mathrm{~m}$, diminuiu o teor de magnésio e fósforo na profundidade 0,05 $0,10 \mathrm{~m}$. A acidez ativa do solo e o teor de potássio diminuíram conforme se aprofunda no perfil solo.

Palavras-chave: Plantio direto e convencional, gessagem, perfil do solo

\begin{abstract}
The combination of gypsum application and soil management may influence soil chemical properties. In this context, the aim of this paper was to evaluate soil chemical properties under two management systems and residual gypsum in soil after 55 months from treatment application. This experiment was developed in FCA/UFGD in Dourados-MS, Brazil under a randomized block design with subdivided plots and four replications. Main plots, subplots and subsubplots consisted of tillage systems (conventional and no tillage), gypsum application and soil depth $(0-0,05,0,05-0,10,0,10-0,15,0,15-0,20$ and $0,20-0,30$ $\mathrm{m})$, respectively. There were no effects of residual gypsum on calcium levels. The combination of no tillage system and residual gypsum increased potassium and sulfur as well as reduced potential acidity.

\footnotetext{
1 Prof. Adjunto do Centro Universitário da Grande Dourados, UNIGRAN, Dourados, MS. E-mail: fabio.souza@unigran.br

2 Profs. Associado(a) II da Universidade Federal da Grande Dourados, UFGD, Dourados, MS. E-mail: jjr.jr@ufgd.edu.br; yararosa@ufgd.edu.br

$3 \mathrm{Eng}^{\mathrm{O}} \mathrm{Agr}^{\mathrm{O}}$, Dr. Embrapa Agropecuária Oeste, Dourados, MS. E-mail: fietz@cpao.embrapa.br

4 Prof. da Universidade Federal do Amazonas, IEAA/UFAM, Humaitá, AM. E-mail: andersonbergamin@hotmail.com

5 Prof. Assistente I do Dept ${ }^{\circ}$ de Estatística da Universidade Federal do Paraná, UFPR, Curitiba, PR. E-mail: walmes@ufpr.br

* Autor para correspondência
} 
However, residual gypsum increased soil potential acidity in conventional system. No tillage system increased calcium levels in the depths 0-0.05 and 0.05-0.10 m, organic matter down to $0.05 \mathrm{~m}$, sulfur accumulation in the depths $0.05-0.10$ and $0.10-0.15 \mathrm{~m}$, sum of bases and base saturation in the depth 0.05-0.10. Conversely, magnesium levels in the depth $0.10-0.15 \mathrm{~m}$, phosphorus from 0.05 to $0.15 \mathrm{~m}$, potential acidity from 0.05 to $0.10 \mathrm{~m}$ and ECC in the depth $0.10-0.15 \mathrm{~m}$ were decreased. Effects of residual gypsum increased sulfur and base saturation from 0.20 to $0.30 \mathrm{~m}$, but decreased magnesium and phosphorus levels in the depth $0.05-0.10 \mathrm{~m}$. Soil active acidity and potassium levels were decreased in deeper soil layers.

Key words: No tillage and tillage, gypsum application, soil profile

\section{Introdução}

O Brasil figura entre as maiores potências na produção de grãos no mundo, cultivando aproximadamente 47.493,3 milhões de hectares, dos quais a região Centro-Oeste representa 14.884,8 milhões de hectares (CONAB, 2009). Grande parte dos solos cultivados dessa região situa-se no bioma do cerrado, apresentando boas características físicas, porém limitação das propriedades químicas, como acidez alta e baixa fertilidade.

Segundo Calegari et al. (2006)áreas intensamente cultivadas através do sistema convencional de produção, com revolvimento do solo e incorporação dos resíduos vegetais, tem contribuído para o processo de degradação das propriedades físicas, químicas e biológicas do solo.

Diante disso a comunidade científica tem intensificado suas pesquisas com o anseio de diagnosticar, elucidar e sugerir correções, adubações e manejos que viabilizem e promovem maximização econômica e produtiva. Dentre essas práticas, o plantio direto é um dos que mais repercutiu no setor agrícola, trazendo mudanças em máquinas, implementos e na concepção dos agricultores em relação à produção sustentável.

O plantio direto reduz a incidência de plantas indesejáveis, cria condições desejáveis a germinação, melhora a agregação do solo proporcionado melhor infiltração de água e maiores condutividades hidráulicas (LLANILLO et al., 2006), estimula a mineralização da matéria orgânica liberando nutrientes (TRIPLETT; DICK, 2008), Naramabuye e Haynes (2006) comentam que o plantio direto, devido a presença de matéria orgânica, aumenta o $\mathrm{pH}$ do solo e reduz a toxicidade por alumínio.

Para alteração e elevação do $\mathrm{pH}$ dos solos no Brasil é comum o uso de calcário o qual segundo Caires et al. (2003), pode ter sua ação limitada ao local de aplicação, não tendo efeito rápido na redução da acidez de camadas mais profundas, que dependem da lixiviação de carbonatos através do perfil do solo. O tempo de reação da calagem pode variar em função da dose aplicada, (CAIRES, 2000). Os autores discorrem ainda no tocante ao calcário aplicado na superfície, o qual apresentou efeito mais lento, promovendo aumento do $\mathrm{pH}$, cálcio, magnésio e saturação por bases e maior redução na acidez potencial somente após 28 meses. A acidez potencial é função do conteúdo de $\left(\mathrm{H}+\mathrm{Al}^{3+}\right)$, e essa pode influenciar na disponibilidade de alguns nutrientes e na atividade microbiana. O alumínio é o principal elemento associado ao efeito negativo da acidez do solo sobre as plantas (RAIJ, 2008).

Vários autores (PAVAN; BINGHAM; PRATT, 1984; OATES; CALDWELL, 1985; ALVA et al., 1986; FARINA; CHANNON 1988; MARSH; GROVE, 1992; CAIRES et al., 1999, CAIRES; FELDAHAUS; BLUM, 2001, CAIRES et al., 2003; CAIRES et al., 2004; CAIRES et al., 2006; SORATTO; CRUSCIOL, 2008; RAIJ, 2008), têm observado o efeito do gesso na redução dos teores de $\mathrm{Al}$ do solo.

O emprego do gesso agrícola tem inúmeras utilizações, destacando-se o efeito fertilizante pelo fornecimento de cálcio e enxofre, efeito na redução da sodicidade, condicionador de 
superfície, condicionador de estercos e preventivo de enfermidades de plantas (VITTI et al., 2008). A relação gesso agrícola e calcário pode compensar o efeito reduzido do calcário apenas no local de aplicação, pois o gesso agrícola atuará no subsolo, nos primeiros anos de cultivo, sem necessidade de incorporação prévia (CAIRES et al., 2003).

Favaretto et al. (2008), estudaram os efeitos de gesso em um solo cultivado com milho e observaram que a adição de gesso ao solo diminuiu a lixiviação de fósforo e potássio e aumentou a concentração de cálcio. Entretanto, são escassos os estudos sobre os efeitos, nas propriedades químicas do solo, da aplicação de gesso em plantio direto e plantio convencional após período razoavelmente longo de implantação dos sistemas de manejo.

Este trabalho foi realizado com os objetivos de avaliar os atributos químicos do solo submetido a dois manejos do solo e efeito residual de gesso, após 55 meses de implantação dos tratamentos.

\section{Material e Métodos}

O trabalho foi conduzido em uma área do aeroporto municipal de Dourados-MS, cedida em regime de comodato a UFGD, Localizado nas coordenadas $22^{\circ} 12^{\prime} 42,74^{\prime}$ ' S e $54^{\circ} 56^{\prime} 17,26^{\prime}$ 'W, com altitude de 452 metros. O experimento foi implantado em outubro de 2003 em um Latossolo Vermelho distroférrico, originalmente sob vegetação de cerrado. O histórico revelou que a área em estudo, nunca havia sido cultivada anteriormente, cuja vegetação predominante é de gramíneas. A vegetação natural foi inicialmente incorporada ao solo com aração e grade pesada.

Este experimento foi implantado com a finalidade de ser de longa duração, portanto foram realizadas as correções da fertilidade necessárias antes de iniciar a semeadura das culturas, visto que após o estabelecimento do sistema plantio direto, as futuras correções serão feitas na superfície do solo, sem incorporação. No mês de outubro de 2003, após o preparo inicial do solo, foi realizada amostragem do mesmo, na profundidade de $0-20 \mathrm{~cm}$, que apresentou a seguinte fertilidade: $\mathrm{pH}$ em $\mathrm{CaCl}_{2}(4,2) ; \mathrm{Al}(1,12$ cmolc dm $\left.{ }^{-3}\right) ; \mathrm{H}+\mathrm{Al} \mathrm{10,5}\left(\right.$ cmolc dm$\left.^{-3}\right) ; \mathrm{P}(2,0 \mathrm{mg}$ $\left.\mathrm{dm}^{-3}\right)$; K (0,2 cmolc dm $\left.{ }^{-3}\right)$; Ca $\left(2,16 \mathrm{cmolc} \mathrm{dm}^{-3}\right)$; $\operatorname{Mg}\left(1,7 \mathrm{cmolc} \mathrm{dm}^{-3}\right) ; \mathrm{S}\left(5,5 \mathrm{mg} \mathrm{dm}^{-3}\right)$ e saturação de bases (28\%). A dose de calcário aplicada em toda a área experimental foi determinada a partir da incubação prévia do solo, para elevar o $\mathrm{pH}$ original do solo a 6,0. O ajuste da curva de regressão indicou uma dose de 4,5 toneladas de calcário por hectare. $\mathrm{Na}$ calagem foi utilizado o calcário dolomítico com 75\% de PRNT, dividido em duas aplicações, sendo a metade da dose incorporada com arado e a outra metade com grade.

Para coleta das amostras de solo foram abertas trincheiras, com dimensões de $50 \mathrm{~cm}$ de largura por $50 \mathrm{~cm}$ de comprimento e com $40 \mathrm{~cm}$ de profundidade.

Para determinação dos atributos químicos do solo foram coletas três amostras de solo por profundidade na entre linha da cultura da soja após a colheita, totalizando 12 repetições por tratamento, em cada profundidade. As profundidades analisadas $0-0,05$, $0,05-0,10,0,10-0,15,0,15-0,20$ e $0,20-0,30 \mathrm{~m}$.

As propriedades químicas estudadas foram $\mathrm{pH}$ em água, em KCl 1 mol. $\mathrm{L}^{-1}$ e em $\mathrm{CaCl}_{2}$ 0,01 mol. $\mathrm{L}^{-1}$, determinados seguindo metodologias descritas por (EMBRAPA, 1997). O Alumínio, o Cálcio e o Magnésio trocáveis foram obtidos segundo metodologia descrita por Defelipo e Ribeiro (1981), e determinou-se o potássio trocável pelo método proposto por Vettori (1969), sendo sua determinação obtida por fotometria de chama.

A matéria orgânica do solo foi determinada segundo Raij e Quaggio (1983). Obteve-se o fósforo pela extração de sua forma disponível seguindo o procedimento descrito por Defelipo e Ribeiro (1981), sendo que para sua determinação utilizou-se fotocalorímetro, usando-se solução sulfomobilítica, conforme recomendado por (BRAGA; DEFELIPO, 1974). Acidez trocável determinada de acordo com 
o método descrito por (EMBRAPA, 1997). Soma de base e CTC foram mensurado usando a descrição segundo Embrapa (1997), Saturação por bases (V\%), foi calculada com uso da formula $\mathrm{V}=100$. SB/T. O enxofre foi analisado com o extrator fosfato monocálcico em água onde avalia o enxofre prontamente solúvel e parte do $\mathrm{S}_{-} \mathrm{SO}_{4}^{-2}$ adsorvido (ALVAREZ, et al., (2001).

O experimento foi disposto no delineamento de blocos casualizados com os fatores arranjados em parcela subsubdividida. As parcelas (sistemas de manejo), sub-parcelas (gessagem) e subsubparcelas (profundidades) com quatro repetições. Os sistemas de manejo estudados foram plantio direto e plantio convencional, cruzados com o efeito residual da aplicação de gesso agrícola na dose de $2000 \mathrm{~kg} \mathrm{ha}^{-1}$ e a ausência de aplicação, cruzados com as profundidades de amostragem que foram de $0-0,05,0,05-0,10,0,10-0,15,0,15-0,20$ e $0,20-0,30$ $\mathrm{m}$ no perfil do solo, perfazendo as 20 combinações possíveis entre os níveis dos fatores.

No tratamento com gesso, o mesmo foi aplicado a lanço e incorporado ao solo com grade niveladora, na primeira quinzena de outubro de 2003. Realizouse ainda a correção para fósforo e potássio, que foram feitas aplicando-se a lanço em toda a área experimental $150 \mathrm{~kg} \mathrm{ha}^{-1}$ de $\mathrm{P}_{2} \mathrm{O}_{5}$, tendo como fonte o fosfato monoamônio (MAP), $100 \mathrm{~kg} \mathrm{ha}^{-1} \mathrm{de}$ $\mathrm{K}_{2} \mathrm{O}$, tendo como fonte o cloreto de potássio. Esses insumos foram aplicados após a primeira operação de incorporação de calcário, em novembro de 2003, e incorporados juntamente com a segunda operação de incorporação de calagem. No sistema de manejo adotado com plantio convencional as operações consistiram em uso de uma grade intermediaria para o preparo primário e de uma grade niveladora, para o preparo secundário, isso ocorria sempre antes da semeadura da cultura. A fórmula utilizada para a adubação da soja foi NPK 00-90-90 respectivamente.

O modelo estatístico para o experimento considerou o efeito de blocos sendo aleatório. A estimativa dos componentes de variância associados aos efeitos aleatórios resultantes foram obtidos pelo método da máxima verossimilhança restrita (ou residual, REML) (SEARLE; CASELLA; MUCCLLOCH, 1992).

Com esse método, considerou-se a estrutura de correlação existente entre as medidas repetidas no mesmo indivíduo. A estrutura de covariância usada para as medidas repetidas foi a espacial gaussiana (SCHABENBERGER; PEARCE, 2002). As análises foram realizadas no SAS 8,0 por meio do PROC MIXED. Os testes F para respostas aos tratamentos foram aplicados segundo aproximação de (SATTERTHWAITE, 1946). Analises de variâncias preliminares foram realizadas pelo PROC GLM no qual foi procedido o teste de Bartlett (1937) para homocedasticidade. Para as variáveis em que a hipótese de homocedasticidade foi rejeitada aplicou-se a transformação de Box e Cox (1964) para a estabilização da variância. Uma vez que a variável alumínio não satisfez as pressuposições de homocedasticidade, nem mesmo por meio de transformação de dados, optou-se pela modelagem estatística por meio da regressão logística, pertencente à classe de modelos lineares generalizados.

Tal procedimento considerou que a distribuição condicional do número ocorrências de alumínio ao longo das repetições é binomial sendo sua média função dos fatores em estudo. A dicotomização do nível de alumínio foi de acordo com o valor de referência $0,05 \mathrm{mmol} \mathrm{dm}^{-3}$ que representa o nível de alumínio proposto, assumindo valor 0 se menor e valor 1 se maior ou igual ao valor de referência. Apenas os efeitos simples dos fatores sistema e gesso foram considerados, sendo a profundidade tratada como uma variável regressora.

\section{Resultados e Discussão}

Após 55 meses de implantação dos sistemas de manejo e aplicação de gesso observou-se que houve efeito significativo para todos os atributos 
químicos do solo uns para interação entre manejo do solo e gessagem, outros entre manejo do solo e profundidade, entre gessagem e profundidade e ainda somente efeito da profundidade.
Houve efeito significativo para as interações entre manejos do solo e efeito residual de gesso para o variável potássio $\left(\mathrm{K}^{+}\right)$, matéria orgânica (MO), enxofre $\left(\mathrm{S}_{-} \mathrm{SO}_{4}{ }^{2-}\right)$, acidez potencial $(\mathrm{H}+\mathrm{Al})$ e capacidade de troca de cátions (CTC) (Tabela 1).

Tabela 1. Desdobramento da interação entre sistemas de manejo e efeito residual de gesso.

\begin{tabular}{|c|c|c|c|c|c|c|}
\hline \multirow[b]{3}{*}{ Gesso } & \multicolumn{2}{|c|}{$\mathrm{K}^{+}$} & \multicolumn{2}{|c|}{ MO } & \multicolumn{2}{|c|}{$\mathrm{S}-\mathrm{SO}_{4}^{2-}$} \\
\hline & \multicolumn{2}{|c|}{$\mathrm{mmol}_{\mathrm{c}} \mathrm{dm}^{-3}$} & \multicolumn{2}{|c|}{$\mathrm{g} \mathrm{dm}^{-3}$} & \multicolumn{2}{|c|}{$\mathrm{mg} \mathrm{dm} \mathrm{dm}^{-3}$} \\
\hline & PC & PD & $\mathrm{PC}$ & PD & PC & PD \\
\hline 0 kg.ha- ${ }^{-1}$ & $3,62 \mathrm{Aa}$ & $2,21 \mathrm{Bb}$ & $29,50 \mathrm{Aa}$ & $32,73 \mathrm{Aa}$ & $12,29 \mathrm{Aa}$ & $11,39 \mathrm{Ab}$ \\
\hline \multirow[t]{3}{*}{$2000 \mathrm{~kg} \cdot \mathrm{ha}^{-1}$} & $3,25 \mathrm{Aa}$ & $3,97 \mathrm{Aa}$ & $29,80 \mathrm{Aa}$ & $28,84 \mathrm{Ab}$ & $30,03 \mathrm{Ba}$ & $37,31 \mathrm{Aa}$ \\
\hline & \multicolumn{3}{|c|}{ Acidez potencial $(\mathrm{H}+\mathrm{Al})$} & \multicolumn{3}{|c|}{ CTC } \\
\hline & \multicolumn{3}{|c|}{$\mathrm{mmol}_{\mathrm{c}} \mathrm{dm}^{-3}$} & \multicolumn{3}{|c|}{$\mathrm{mmol}_{\mathrm{c}} \mathrm{dm}^{-3}$} \\
\hline Gesso & PC & & PD & & & $\mathrm{PD}$ \\
\hline 0 kg.ha- ${ }^{-1}$ & $53,85 \mathrm{Aa}$ & & $52,15 \mathrm{Aa}$ & 121, & & $127,72 \mathrm{Aa}$ \\
\hline $2000 \mathrm{~kg} \cdot \mathrm{ha}^{-1}$ & $55,80 \mathrm{Aa}$ & & $45,15 \mathrm{Bb}$ & 124, & & $114,00 \mathrm{Ab}$ \\
\hline
\end{tabular}

Médias seguidas de letras maiúsculas iguais nas linhas e letras minúsculas iguais nas colunas não diferem entre si pelo teste de Tukey ao nível de 5\%. PD - Plantio direto e PC - Plantio Convencional.

Fonte: Elaboração dos autores.

O plantio convencional promoveu maior acúmulo de $\mathrm{K}^{+}$, em relação ao plantio direto ambos na ausência de efeito residual de gesso (Tabela 1), porém esses sistemas não diferiram entre si na presença de efeito residual de gesso. O menor acúmulo obtido com o plantio direto na ausência de gesso é contrário ao citado por Raij (2008), onde o autor ressalta a preocupação em relação às gessagens elevadas que podem remover do solo o $\mathrm{K}^{+}$, lixiviando este nutriente. Farina, Channon e Thibaud (2000), não constataram efeito do gesso na remoção de $\mathrm{K}^{+}$.

A matéria orgânica do solo (Tabela 1), apenas apresentou diferença entre a presença e ausência do gesso no sistema de plantio direto, onde o menores valores são verificados na presença do gesso. Este fato pode ser devido ação do gesso no solo e, consequentemente, na atividade microbiana, pois ao lixiviar no perfil do solo o gesso favorece o aumento de teores de cálcio e redução dos teores de alumínio, promovendo maior aprofundamento das raízes, as quais distribuem-se melhor no perfil do solo propiciando a mineralização mais profunda e acelerada.

$\mathrm{Na}$ Tabela 1, o teor de $\mathrm{S}_{-} \mathrm{SO}_{4}{ }^{2-}$ foi superior em ambos manejos que receberam o gesso, enquanto que se comparado a média de cada sistema tomando-se as médias dos níveis de gesso, o plantio direto promoveu maior acumulo de $\mathrm{S}_{-} \mathrm{SO}_{4}{ }^{2-}$ . Entretanto, dentro dos sistemas de plantio houve apenas diferença significativa para o efeito do gesso no plantio direto. Essa diferença é devida à concentração de $\mathrm{S}$ na composição do gesso agrícola. Vitti et al. (2008), comentam que a valores de $\mathrm{S}$ em gesso agrícola são de $15 \%$.

A interação plantio direto com efeito de gesso promoveu uma redução na acidez potencial do solo (Tabela 1), Soratto e Crusciol (2008), notaram que após 18 meses o gesso combinado com calcário não promoveram redução da $\mathrm{H}+\mathrm{Al}$, sendo contrario ao observado neste trabalho, onde mesmo 55 meses após a aplicação, o gesso continuou a proporcionar efeito na acidez potencial. 
A capacidade de troca de cátions (CTC) apenas foi influenciada pelo efeito de gesso sob o plantio direto, onde a CTC na presença de gesso foi inferior significativamente. Como a CTC é dependente da quantidade e do tipo de argila e de matéria orgânica, observa-se que a CTC diminui na interação entre plantio direto com efeito residual de gesso, fato verificado também para os teores de matéria orgânica no mesmo tratamento.

Para a variável cálcio $\left(\mathrm{Ca}^{+2}\right)$, magnésio $\left(\mathrm{Mg}^{+2}\right)$, matéria orgânica $(\mathrm{MO})$, fósforo $(\mathrm{P})$, enxofre (S$\mathrm{SO}_{4}^{2-}$ ), acidez potencial $(\mathrm{H}+\mathrm{Al})$, soma de base $(\mathrm{S})$ , CTC e saturação por base (V\%), houve efeito significativo para interação manejos do solo e profundidades (Tabela 2).

Tabela 2. Desdobramento da interação entre os sistemas de manejo e profundidade.

\begin{tabular}{|c|c|c|c|c|c|c|}
\hline \multirow[b]{3}{*}{ Profundidade } & \multirow{2}{*}{\multicolumn{2}{|c|}{$\frac{\mathrm{Ca}^{2+}}{\mathrm{mmol}_{\mathrm{c}} \mathrm{dm}^{-3}}$}} & \multirow{2}{*}{\multicolumn{2}{|c|}{$\frac{\mathrm{Mg}^{2+}}{\mathrm{mmol}_{\mathrm{c}} \mathrm{dm}^{-3}}$}} & \multirow{2}{*}{\multicolumn{2}{|c|}{$\frac{\mathrm{MO}}{\mathrm{g} \mathrm{dm}^{-3}}$}} \\
\hline & & & & & & \\
\hline & $\mathrm{PC}$ & PD & $\mathrm{PC}$ & PD & $\mathrm{PC}$ & PD \\
\hline $0-0,05 \mathrm{~m}$ & $63,01 \mathrm{Ba}$ & $75,55 \mathrm{Ab}$ & $26,25 \mathrm{Aa}$ & $27,35 \mathrm{Aa}$ & $36,21 \mathrm{Ba}$ & $45,93 \mathrm{Aa}$ \\
\hline $0,05-0,10 \mathrm{~m}$ & $64,04 \mathrm{Ba}$ & $88,15 \mathrm{Aa}$ & $25,98 \mathrm{Aa}$ & $28,07 \mathrm{Aa}$ & $34,78 \mathrm{Aa}$ & $34,38 \mathrm{Ab}$ \\
\hline $0,10-0,15 \mathrm{~m}$ & $58,89 \mathrm{Aa}$ & $63,91 \mathrm{Ac}$ & $27,00 \mathrm{Aa}$ & $15,27 \mathrm{Bb}$ & $31,76 \mathrm{Aa}$ & $28,48 \mathrm{Ac}$ \\
\hline $0,15-0,20 \mathrm{~m}$ & $25,93 \mathrm{Ab}$ & $22,89 \mathrm{Ad}$ & $10,37 \mathrm{Ab}$ & 7,80 Ac & $24,53 \mathrm{Ab}$ & $24,16 \mathrm{Acd}$ \\
\hline \multirow[t]{3}{*}{$0,20-0,30 \mathrm{~m}$} & $16,51 \mathrm{Ab}$ & $16,13 \mathrm{Ad}$ & $4,91 \mathrm{Ac}$ & $3,01 \mathrm{Ac}$ & $20,96 \mathrm{Ab}$ & $20,98 \mathrm{Ad}$ \\
\hline & \multicolumn{2}{|c|}{$\mathrm{P}$} & \multicolumn{2}{|c|}{$\mathrm{S}_{-} \mathrm{SO}_{4}^{2-}$} & \multicolumn{2}{|c|}{$\mathrm{H}+\mathrm{Al}$} \\
\hline & \multicolumn{2}{|c|}{$\mathrm{mg} \mathrm{dm}^{-3}$} & \multicolumn{2}{|c|}{$\mathrm{mg} \mathrm{dm^{-3 }}$} & \multicolumn{2}{|c|}{$\mathrm{mmol}_{c} \mathrm{dm}^{-3}$} \\
\hline Profundidade & $\mathrm{PC}$ & PD & $\mathrm{PC}$ & PD & $\mathrm{PC}$ & PD \\
\hline $0-0,05 \mathrm{~m}$ & $23,38 \mathrm{Aa}$ & $24,50 \mathrm{Aa}$ & $2,45 \mathrm{Ab}$ & $4,15 \mathrm{Ab}$ & $39,88 \mathrm{Ac}$ & $31,50 \mathrm{Ac}$ \\
\hline $0,05-0,10 \mathrm{~m}$ & $16,88 \mathrm{Aa}$ & $6,00 \mathrm{Bb}$ & $1,20 \mathrm{Bb}$ & $9,14 \mathrm{Ab}$ & $37,13 \mathrm{Ac}$ & $20,75 \mathrm{Bc}$ \\
\hline $0,10-0,15 \mathrm{~m}$ & $4,75 \mathrm{Ab}$ & $2,13 \mathrm{Bc}$ & $1,45 \mathrm{Bb}$ & $10,25 \mathrm{Ab}$ & $33,38 \mathrm{Ac}$ & $22,88 \mathrm{Ac}$ \\
\hline $0,15-0,20 \mathrm{~m}$ & $2,25 \mathrm{Ac}$ & $1,63 \mathrm{Ac}$ & $44,53 \mathrm{Aa}$ & $42,67 \mathrm{Aa}$ & $73,25 \mathrm{Ab}$ & $74,12 \mathrm{Ab}$ \\
\hline \multirow[t]{3}{*}{$0,20-0,30 \mathrm{~m}$} & $1,50 \mathrm{Ac}$ & $1,00 \mathrm{Ac}$ & $55,35 \mathrm{Aa}$ & $55,52 \mathrm{Aa}$ & $90,50 \mathrm{Aa}$ & $94,00 \mathrm{Aa}$ \\
\hline & \multicolumn{2}{|c|}{ Soma de bases } & \multicolumn{2}{|c|}{ CTC } & \multicolumn{2}{|c|}{$\mathrm{V}(\%)$} \\
\hline & \multicolumn{2}{|c|}{$\mathrm{mmol}_{\mathrm{c}} \mathrm{dm}^{-3}$} & \multicolumn{2}{|c|}{$\mathrm{mmol}_{\mathrm{c}} \mathrm{dm}^{-3}$} & \multicolumn{2}{|c|}{$\%$} \\
\hline Profundidade & $\mathrm{PC}$ & PD & $\mathrm{PC}$ & PD & $\mathrm{PC}$ & $\mathrm{PD}$ \\
\hline $0-0,05 \mathrm{~m}$ & $98,91 \mathrm{Aa}$ & $111,94 \mathrm{Aa}$ & $138,79 \mathrm{Aa}$ & $143,44 \mathrm{Aa}$ & $71,25 \mathrm{Aa}$ & $78,12 \mathrm{Aa}$ \\
\hline $0,05-0,10 \mathrm{~m}$ & $94,03 \mathrm{Ba}$ & $119,55 \mathrm{Aa}$ & $131,15 \mathrm{Aab}$ & $140,30 \mathrm{Aa}$ & $71,63 \mathrm{Ba}$ & $85,00 \mathrm{Aa}$ \\
\hline $0,10-0,15 \mathrm{~m}$ & $87,84 \mathrm{Aa}$ & $80,79 \mathrm{Ab}$ & $121,21 \mathrm{Ab}$ & $103,63 \mathrm{Bb}$ & $72,63 \mathrm{Aa}$ & 77,75 Aa \\
\hline $0,15-0,20 \mathrm{~m}$ & $37,20 \mathrm{Ab}$ & $31,56 \mathrm{Ac}$ & $110,45 \mathrm{Ab}$ & $105,69 \mathrm{Ab}$ & $33,50 \mathrm{Ab}$ & $29,88 \mathrm{Ab}$ \\
\hline $0,20-0,30 \mathrm{~m}$ & $22,06 \mathrm{Ac}$ & $19,74 \mathrm{Ac}$ & $112,56 \mathrm{Ab}$ & $113,74 \mathrm{Ab}$ & $19,63 \mathrm{Ac}$ & $17,75 \mathrm{Ac}$ \\
\hline
\end{tabular}

Médias seguidas de letras maiúsculas iguais nas linhas e letras minúsculas iguais nas colunas não diferem entre si pelo teste de Tukey ao nível de 5\%. PD - Plantio direto e PC - Plantio Convencional.

Fonte: Elaboração dos autores.

O plantio direto acumulou maior teor de $\mathrm{Ca}^{+2}$ nas profundidades $0-0,05$ e $0,05-0,10 \mathrm{~m}$. Farina, Channon e Thibaud (2000) observaram que a aplicação de gesso com calcário promoveu maiores concentrações de Cálcio entre profundidade de 0,15 a $0,30 \mathrm{~m}$, diminuindo conforme se aprofunda no solo. Caíres et al. (2003) trabalhando com aplicação de calcário e gesso na implantação de plantio direto observou que o Cálcio aumentou também em profundidade.

É importante comentar que na implantação dos tratamentos no solo da área experimental o teor de $\mathrm{Ca}^{2+}$ era de 21,6 mmol $\mathrm{dm}^{-3}$ na camada de 0-0,20 m, sendo substancial esse aumento da concentração do $\mathrm{Ca}^{2+}$ em função da aplicação do calcário e gesso. Porém pode haver certa limitação 
imposta por menores valores de cálcio encontrado na profundidade de $0,20-0,30 \mathrm{~cm}$, como cita Vitti et al. (2008), que a menor proporção de $\mathrm{Ca}^{2+}$ em profundidade pode causar restrições ao crescimento radicular, devido a menor troca catiônica entre $\mathrm{Ca}^{2+}$ e $\mathrm{Al}^{3+}$ adsorvido na fração argila.

Para o $\mathrm{Mg}^{2+}$ diferiu significativamente entre os sistemas apenas na profundidade de 0,10-0,15 m (Tabela 2), o plantio convencional mostrou-se superior ao plantio direto apenas na profundidade de $0,10-0,15 \mathrm{~m}$.

Observou-se que o teor de $\mathrm{Mg}^{2+}$ diminui com o aumento da profundidade, sendo que no plantio direto as profundidades 0-0,05 e 0,05-0,10 m tiveram maiores acúmulos desse nutriente e as menores concentrações ocorreram nas profundidades de 0,15-0,20 e 0,20-0,30 m, o plantio convencional seguiu essa mesma tendência. Segundo Santos et al. (2006), os teores de $\mathrm{Mg}^{2+}$ trocável foram afetados pelos sistemas de cultivo apenas na profundidade de 0-0,05 m, sendo o plantio direto superior ao preparo convencional e contrário ao obtido neste trabalho.

A maior concentração de $\mathrm{Mg}^{2+}$ nas profundidades mais superficiais pode ter ocorrido pela aplicação de calcário dolomítico na implantação do experimento.

Sabe-se que um dos benefícios obtidos com o plantio direto é o aumento do teor de MO com o passar do tempo, principalmente nas camadas mais superficiais, isso explica a diferença significativa entre os sistemas de manejo para a profundidade de 0-0,05 m, onde o plantio direto promoveu maior concentração de $\mathrm{MO}$, porém nas demais profundidades não se observaram diferença. Houve um decréscimo no conteúdo de MO em ambos os sistemas conforme se aprofundou no solo.

No plantio direto o maior teor se observa na profundidade de 0-0,05 $\mathrm{m}$ e o menor 0,20-0,30 $\mathrm{m}$, no plantio convencional não diferiram as profundidades de 0-0,05, 0,05-0,10 e 0,10-0,15 m, e essas diferiam significativamente das profundidades de 0,15-0,20 e 0,20-0,30 m. De acordo com Ciotta et al. (2003), o acúmulo de matéria orgânica restringese às camadas superficiais do solo, cuja importância sobre as condições físicas, químicas e biológicas do solo possivelmente não sejam refletidas pelos baixos valores de acúmulo verificados na camada de 0-0,20 $\mathrm{m}$ do solo, na qual o efeito é diluído. Zambrosi, Alleoni e Caires (2007), avaliando a aplicação de calcário combinado com doses de gesso em plantio direto notaram a mesma tendência da matéria orgânico do solo ao diminuir quando se aprofundou no solo.

$\mathrm{Na}$ tabela 2, pode se observar que o plantio convencional foi superior ao plantio direto nas profundidades de 0,05-0,10 e 0,10-0,15 m para variável $\mathrm{P}$, corroborando com os valores obtidos por Oliveira et al. (2004), onde esses autores avaliando um Latossolo Vermelho após 20 anos de cultivo notaram que o teor de $\mathrm{P}$ reduziu na profundidade de 0,10-0,20 $\mathrm{m}$ no sistema de semeadura direta, entretanto discorda dos valores verificados no trabalho de Costa, Goedert e Sousa (2006), em que tais pesquisadores observaram diferença entre os sistemas de manejo após longo período de cultivo, sendo que o plantio direto após 8 anos de cultivo acumulou maior teor de $\mathrm{P}$ na profundidade de $0-0,10$ $\mathrm{m}$ não diferindo nas camadas subseqüentes.

Provavelmente esse maior teor significativo de $\mathrm{P}$ nas profundidades de $0,05-0,10$ e $0,10-0,15 \mathrm{~m}$ para o plantio convencional é devido ao revolvimento do solo neste sistema, pois, como o fósforo tem a menor mobilidade entre os nutrientes, e este foi aplicado a lanço na implantação dos sistemas formando banco de nutriente e posteriormente nas safras adubadas logo abaixo da linha de semeadura, com a ocorrência de gradagens freqüentes nas safras de 2003/2004, 2004/2005, 2005/2006, 2007/2008, o fósforo acabou sendo incorporado em profundidade onde há ação da grade intermediária e niveladora.

Para a variável $\mathrm{S}_{-} \mathrm{SO}_{4}{ }^{2-}$ o efeito pronunciável aconteceu com o uso de plantio direto (Tabela 2) apenas nas profundidades de 0,05-0,10 e 0,10$0,15 \mathrm{~m}$.

Esse fato possivelmente é devido aos teores de matéria orgânica verificados nas profundidades mais superficiais, e como o $\mathrm{S}_{-} \mathrm{SO}_{4}{ }^{2-}$ não é atraído 
para a superfície dos colóides e da matéria orgânica, ele pode ser removido com a água para camadas mais profundas do solo, então esse maior conteúdo de $\mathrm{S}_{-} \mathrm{SO}_{4}{ }^{2-}$ nessas profundidades pode ter acontecido pela transformação biológica da mineralização da matéria orgânica, liberando sulfatos e composto de sulfato para o solo. Essa explicação cabe também a maior concentração deste nutriente nas profundidades de 0,15-0,20 e 0,20-0,30 m. Nziguheba, Smolders e Merckx (2005), comentam que mineralização e imobilização regulam o ciclo no solo e controlam a disponibilidade de $\mathrm{S}$ às plantas.

O plantio direto provocou um decréscimo na $\mathrm{H}+\mathrm{Al}$ somente na profundidade de 0,05-0,10 $\mathrm{m}$ (Tabela 2) quando comparado com o plantio convencional, corroborando com Soratto e Crusciol (2008) que após 18 meses observaram desempenho igual na ausência de gessagem na implantação do sistema de plantio direto.

Avaliando o $\mathrm{H}+\mathrm{Al}$ nas profundidades em ambos os sistemas, ocorreu aumento do $\mathrm{H}+\mathrm{Al}$ nas profundidades de 0,15-0,20 e 0,20-0,30 cm. Como a acidez potencial do solo, quanto mais elevada, maior será a quantidade de íons $\mathrm{H}^{+}$e $\mathrm{Al}^{+3}$ que poderão vir para a solução. Considerando que o aumento da acidez potencial em profundidade limita a expansão do sistema radicular, dificultando assim o acesso a água e aos nutrientes que se encontram nas camadas mais profundas do solo.

A soma de bases foi diferente significativamente entre os sistemas apenas na profundidade de 0,05-0,10 m (Tabela 2), sendo inferior no plantio convencional, isto pode ser em função da menor acidez potencial obtida nesta camada de solo. As profundidades diferiram entre si, as maiores concentrações de soma de bases encontram-se nas camadas mais superficiais em ambos os sistemas, diminuindo conforme se aprofunda no perfil do solo. Costa et al. (2007), analisando o efeito de sistemas de manejos e gesso não observaram diferença entre os manejos para soma de bases.

O plantio convencional foi superior ao plantio direto para CTC somente na profundidade de 0,10-
0,15 m (Tabela 2). Hussain, Olson e Ebelhar (1999) obtiveram em sua pesquisa diferença significativa entre os sistemas de manejo na profundidade de 0-0,05 m favorável ao plantio direto, resultado que discorda dos valores verificados neste trabalho.

Diferenças entre as profundidades apontaram redução na CTC do solo nas maiores profundidades, entretanto em ambos os sistemas a CTC ainda permanece em condições satisfatórias para o cultivo de plantas, considerando que a CTC indica a capacidade total de retenção de cátions, os quais, em geral, irão tornar-se disponíveis às plantas.

Entre os manejos houve diferença significativa para V\% somente na profundidade de 0,05-0,10 $\mathrm{m}$, sendo mais proeminente para o plantio direto. Houve diferença para as profundidades, sendo que nos dois sistemas os valores de $\mathrm{V} \%$ diminuíram à medida que se aprofundou no perfil do solo.

Cavalieri et al. (2004), verificaram a mesma tendência para o sistema de plantio direto, decrescendo com maiores profundidades, ressalvase ainda que o valor obtido neste trabalho até a profundidade de $0,15 \mathrm{~m}$ encontra-se dentro do recomendável para a maioria das culturas que varia entre 50 a $60 \%$, por este motivo convêm no momento da coleta do solo estratificar mais as amostras de solo para não superestimar valores de saturação por base, observando que logo abaixo da profundidade $0,15 \mathrm{~m}$, os valores de saturação por base diminuem drasticamente.

$\mathrm{Na}$ Tabela 3 são apresentados os resultados dos testes de médias sob a interação entre efeito residual de gesso e profundidade para variáveis Magnésio $\left(\mathrm{Mg}^{2+}\right)$ acidez potencial $(\mathrm{H}+\mathrm{Al})$, fósforo(P), enxofre $\left(\mathrm{S}_{-} \mathrm{SO}_{4}{ }^{2-}\right)$, soma de bases e saturação por base $(\mathrm{V} \%)$. O gesso, porém, não promoveu diferença para o cálcio como era de se esperar. O argumento para este acontecimento é que possivelmente a correção realizada com o calcário, ocupando as cargas dos colóides, fez com o que o cálcio fosse lixiviado a camadas abaixo das estudadas neste trabalho. 
Tabela 3. Desdobramento da interação entre o efeito residual de gesso e profundidade.

\begin{tabular}{|c|c|c|c|c|c|c|}
\hline \multirow[b]{3}{*}{ Profundidade } & \multirow{2}{*}{\multicolumn{2}{|c|}{$\begin{array}{c}\mathrm{Mg}^{2+} \\
\mathrm{mmol}_{\mathrm{c}} \mathrm{dm}^{-3}\end{array}$}} & \multirow{2}{*}{\multicolumn{2}{|c|}{$\frac{\mathrm{H}+\mathrm{Al}}{\mathrm{mmol}_{c} \mathrm{dm}^{-3}}$}} & \multirow{2}{*}{\multicolumn{2}{|c|}{$\frac{\mathrm{P}}{\mathrm{mg} \mathrm{dm}}$}} \\
\hline & & & & & & \\
\hline & 0 kg.ha-1 & 2000 kg.ha ${ }^{-1}$ & 0 kg.ha ${ }^{-1}$ & 2000 kg.ha- ${ }^{-1}$ & 0 kg.ha-1 & 2000 kg.ha ${ }^{-1}$ \\
\hline $0-0,05 \mathrm{~m}$ & $28,91 \mathrm{Ab}$ & $24,69 \mathrm{Aa}$ & $34,75 \mathrm{Ac}$ & $36,63 \mathrm{Ac}$ & $21,50 \mathrm{Aa}$ & $26,38 \mathrm{Aa}$ \\
\hline $0,05-0,10 \mathrm{~m}$ & $30,06 \mathrm{Aa}$ & $23,99 \mathrm{Ba}$ & $24,75 \mathrm{Ac}$ & $33,13 \mathrm{Ac}$ & $14,63 \mathrm{Ab}$ & $8,25 \mathrm{Bb}$ \\
\hline $0,10-0,15 \mathrm{~m}$ & $25,10 \mathrm{Ab}$ & $17,18 \mathrm{Bb}$ & $29,25 \mathrm{Ac}$ & $27,00 \mathrm{Ac}$ & 4,13 Ac & $2,75 \mathrm{Ac}$ \\
\hline $0,15-0,20 \mathrm{~m}$ & $9,14 \mathrm{Ac}$ & $9,04 \mathrm{Ac}$ & $76,25 \mathrm{Ab}$ & $71,12 \mathrm{Ab}$ & $1,50 \mathrm{Ad}$ & 2,38 Acd \\
\hline \multirow[t]{3}{*}{$0,20-0,30 \mathrm{~m}$} & 2,97 Ad & $4,95 \mathrm{Ac}$ & $100,00 \mathrm{Aa}$ & $84,50 \mathrm{Ba}$ & $1,24 \mathrm{Ad}$ & $1,25 \mathrm{Ad}$ \\
\hline & \multicolumn{2}{|c|}{$\mathrm{S}-\mathrm{SO}_{4}^{2-}$} & \multicolumn{2}{|c|}{ Soma de bases } & \multicolumn{2}{|c|}{$\mathrm{V}(\%)$} \\
\hline & \multicolumn{2}{|c|}{$\mathrm{mg} \mathrm{dm}^{-3}$} & \multicolumn{2}{|c|}{$\mathrm{mmol}_{c} \mathrm{dm}^{-3}$} & \multicolumn{2}{|c|}{$\%$} \\
\hline Profundidade & 0 kg.ha-1 & $2000 \mathrm{~kg} \cdot \mathrm{ha}^{-1}$ & 0 kg.ha ${ }^{-1}$ & 2000 kg.ha- ${ }^{-1}$ & 0 kg.ha-1 & 2000 kg.ha ${ }^{-1}$ \\
\hline $0-0,05 \mathrm{~m}$ & $4,53 \mathrm{Ab}$ & $2,08 \mathrm{Ab}$ & $106,94 \mathrm{Aa}$ & $103,91 \mathrm{Aa}$ & $75,50 \mathrm{Aa}$ & $73,87 \mathrm{Aa}$ \\
\hline $0,05-0,10 \mathrm{~m}$ & $4,15 \mathrm{Ab}$ & $6,99 \mathrm{Ab}$ & $109,91 \mathrm{Aa}$ & $103,66 \mathrm{Aa}$ & $81,38 \mathrm{Aa}$ & $75,25 \mathrm{Aa}$ \\
\hline $0,10-0,15 \mathrm{~m}$ & $6,02 \mathrm{Ab}$ & $5,68 \mathrm{Ab}$ & $89,69 \mathrm{Ab}$ & $78,94 \mathrm{Ab}$ & $75,38 \mathrm{Aa}$ & $75,00 \mathrm{Aa}$ \\
\hline $0,15-0,20 \mathrm{~m}$ & $34,25 \mathrm{Aa}$ & $52,95 \mathrm{Aa}$ & $34,89 \mathrm{Ac}$ & $33,88 \mathrm{Ac}$ & $31,13 \mathrm{Ab}$ & $32,25 \mathrm{Ab}$ \\
\hline $0,20-0,30 \mathrm{~m}$ & $10,28 \mathrm{Bb}$ & $100,64 \mathrm{Aa}$ & $16,04 \mathrm{Ad}$ & $25,76 \mathrm{Ac}$ & $13,88 \mathrm{Bc}$ & $23,50 \mathrm{Ac}$ \\
\hline
\end{tabular}

Médias seguidas de letras maiúsculas iguais nas linhas e letras minúsculas iguais nas colunas não diferem entre si pelo teste de Tukey ao nível de 5\%.

Fonte: Elaboração dos autores.

O Magnésio diferiu entre o efeito residual de gesso nas profundidades de 0,05-0,10 e 0,10-0,15 $\mathrm{m}$, sendo inferior na presença do gesso concordando com Caires et al. (2002).

Nas profundidades sob ausência de gesso o maior valor observado foi na profundidade de 0,05-0,10 m e o menor de 0,20-0,30 m (Tabela 3), já na presença residual de gesso o teor de $\mathrm{Mg}^{2+}$ decresceram conforme se aprofundou no perfil do solo, discordando dos resultados apresentados por Ernani et al. (2001), os autores ressalvam que gesso promoveu grande lixiviação de $\mathrm{Mg}^{2+}$ e a magnitude foi maior quando se incorporou o gesso agrícola. Possivelmente a diferença gerada entre a presença e ausência do resíduo de gesso para o $\mathrm{Mg}^{2+}$ esta no fato da gessagem remover este nutriente para camadas mais profundas do solo como relatou (RAIJ, 2008).

Pela tabela 3, pode se notar que mesmo não promovendo diferença significativa entre ausência e a presença, o $\mathrm{Mg}^{2+}$ distribui-se equitativamente na presença residual de gesso. Toma et al. (1999) avaliando o efeito da aplicação de gesso nas propriedades químicas observaram redução do
$\mathrm{Mg}^{2+}$ nas profundidades que compreende entre 0,30 a $0,50 \mathrm{~m}$.

A presença de gesso na profundidade de 0,200,30 m causou redução na acidez potencial do solo (Tabela 3). Estes resultados refletem a influência do gesso sobre os valores de $\mathrm{Al}$ sendo conseqüência provocada pelo sulfato por troca de ligantes, removendo hidroxilas e estas contribuíram para a insolubilização do $\mathrm{Al}$, apesar de ser uma reação termodinâmica instável, cumpre seu papel de reduzir o Al no solo, mesmo que não por muito tempo (RAIJ, 2008).

Analisando o efeito entre as profundidades a acidez potencial aumentou aprofundando-se no solo, tanto na presença como na ausência de efeito residual de gesso. Soratto e Crusciol (2008), após 18 meses da aplicação de gesso observaram efeito na acidez potencial do solo na profundidade que compreende entre 0,40 a 0,60 m. É importante considerar que na implantação dos tratamentos a concentração de $\mathrm{H}+\mathrm{Al}$ era de $105 \mathrm{mmol} \mathrm{dm}^{-3}$, mesmo após 55 meses da aplicação de gesso, principalmente na camada arável reduziu-se satisfatoriamente a acidez potencial. 
Os valores de fósforo (Tabela 3), apontam que o efeito residual de gesso foi inferior somente na profundidade de 0,05-0,10 $\mathrm{m}$ a favor da ausência de gesso. Ao analisarem-se as profundidades dentro da presença e ausência de efeito residual de gesso, ambas comportaram-se de forma semelhante, diminuindo o teor de $\mathrm{P}$ em profundidades maiores.

Estes resultados são contrários aos obtidos por Costa et al. (2007), que avaliando o efeito do plantio direto e plantio convencional com ou sem gesso num Latossolo Vermelho distroférrico, evidenciaram não haver efeito do residual de gesso. Caires et al. (2003) obtiveram aumento linear nos teores de $\mathrm{P}$ na camada superficial do solo 0-0,05 m, no período de 3 anos de cultivo, e os autores atribuíram esse aumento ao $\mathrm{P}$ contido na composição do gesso agrícola.

$\mathrm{O}$ menor teor de $\mathrm{P}$ obtido na profundidade de 0,05-0,10 m, pode ser atribuído ao composto formado entre o Cálcio e o Fósforo, devido a dissociação entre o cálcio e o enxofre na presença

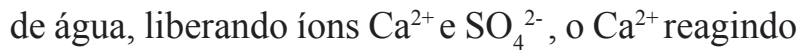
com o $\mathrm{P}$ provocando menor solubilidade e podendo ainda com a formação de fosfato de cálcio causar precipitação do fosfato adicionado ao solo.

Vitti et al. (2008), comentam que um solo quando corrigido sua acidez na camada arável com calcário, permite que o sulfato oriundo do gesso possa se movimentar para camadas inferiores acompanhado por cátions, especialmente cálcio. Isso pode ser observado pela tabela 3 , onde nas camadas mais profundas houve acumulo de $\mathrm{S}-\mathrm{SO}_{4}{ }^{2-}$ com aplicação de gesso, promovendo diferença entre a presença e ausência de efeito residual de gesso para as profundidades de 0,20-0,30 m. O trabalho de Soratto e Crusciol (2008) não corrobora com este trabalho, pois estes autores não verificaram efeito do gesso na concentração de $\mathrm{S}-\mathrm{SO}_{4}{ }^{2-}$ nas após 18 meses de aplicação, creditando esse fato a lixiviação desse nutriente a camadas mais profundas. Similar ao observado neste trabalho foi alcançado na pesquisa de Toma et al. (1999), estes autores demonstraram que o $\mathrm{S}_{-} \mathrm{SO}_{4}{ }^{2-}$ acumulou mais nas profundidades entre 0,30 a $0,60 \mathrm{~m}$.
A soma de bases não diferiu significativamente entre a presença e ausência do efeito residual de gesso, alterando apenas entre as profundidades, ocorrendo redução das bases com o aprofundamento no perfil do solo. Como a soma de bases é função da presença de magnésio, cálcio e potássio, e estes dois últimos não promulgaram efeito significativo com a aplicação de gesso, a soma de bases pode ter sido influenciada por este motivo.

$\mathrm{O}$ efeito de gesso promoveu diferença para $\mathrm{V} \%$ na profundidade de $0,20-0,30 \mathrm{~m}$, sendo obtido maior valor com a presença residual de gesso. Até a profundidade de $0,20 \mathrm{~m}$ a presença e a ausência residual de gesso foram similares tanto entre si e entre as profundidades. Caires et al. (2006) verificaram que após 55 meses de aplicação de gesso a V\% aumentou em camadas abaixo de 0,40 m, concordando com a tendência obtida neste trabalho.

O efeito do gesso em profundidade maior pode ser decorrente do fornecimento de cálcio, promovendo mobilidade em camadas mais profundas do solo, não tendo qualquer relação com a correção da acidez (CAIRES et al., 2002). Soratto e Crusciol (2007) mencionam que este fato foi devido ao movimento

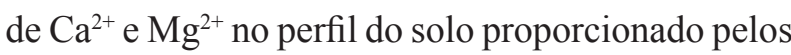
compostos hidrossolúveis originários dos resíduos de culturas antecessoras.

$\mathrm{Na}$ tabela 4, são apresentados os valores de potássio, $\mathrm{pH} \mathrm{CaCl}_{2} \mathrm{pH} \mathrm{H}_{2} \mathrm{O}$ e $\mathrm{pH} \mathrm{KCl}$. Houve efeito significativo apenas para o fator profundidade, não havendo efeito de manejo e residual de gesso.

O teor de potássio decresceu nas camadas mais profundas do solo, sendo a profundidade de 0-0,05 $\mathrm{m}$ que mais acumulou $\mathrm{K}^{+}$, e o menor conteúdo obteve-se na profundidade de 0,20-0,30 m. Thomas, Dalal e Standley (2007) relatam que este acumulo de $\mathrm{K}^{+}$na camada mais superficial do solo, pode afetar negativamente a disponibilidade de potássio às raízes de plantas especialmente quando ocorre seca sazonal, quando o solo seca superficialmente rapidamente. 
Tabela 4. Valores médios de Potássio (K), $\mathrm{pH} \mathrm{CaCl}_{2,} \mathrm{pH} \mathrm{H}_{2} \mathrm{O}$ e $\mathrm{pH} \mathrm{KCl}$ em diferentes profundidades submetido a manejos do solo e efeito residual de gesso.

\begin{tabular}{ccccc}
\hline & & \multicolumn{3}{c}{$\mathrm{pH}$} \\
\cline { 2 - 5 } Profundidade & $\mathrm{K}^{+} \mathrm{mmol}_{\mathrm{c}} \mathrm{dm}^{-3}$ & $\mathrm{CaCl}_{2}$ & $\mathrm{H}_{2} \mathrm{O}$ & $\mathrm{KCl}$ \\
\hline $0-0,05 \mathrm{~m}$ & $9,34 \mathrm{a}$ & $5,34 \mathrm{a}$ & $5,96 \mathrm{a}$ & $5,17 \mathrm{a}$ \\
$0,05-0,10 \mathrm{~m}$ & $3,67 \mathrm{~b}$ & $5,56 \mathrm{a}$ & $6,19 \mathrm{a}$ & $5,41 \mathrm{a}$ \\
$0,10-0,15 \mathrm{~m}$ & $1,78 \mathrm{c}$ & $5,38 \mathrm{a}$ & $6,00 \mathrm{a}$ & $5,14 \mathrm{a}$ \\
$0,15-0,20 \mathrm{~m}$ & $0,89 \mathrm{~d}$ & $4,53 \mathrm{~b}$ & $4,82 \mathrm{~b}$ & $4,20 \mathrm{~b}$ \\
$0,20-0,30 \mathrm{~m}$ & $0,62 \mathrm{e}$ & $4,29 \mathrm{~b}$ & $4,68 \mathrm{~b}$ & $4,11 \mathrm{~b}$ \\
\hline
\end{tabular}

Médias seguidas de letras minúsculas iguais nas colunas não diferem entre si pelo teste de Tukey ao nível de 5\%.

Fonte: Elaboração dos autores.

Apesar de o potássio diminuir quando se aprofunda no subsolo, cabe salientar que na implantação dos tratamentos o teor de $\mathrm{K}^{+}$no solo era de 2,0 $\mathrm{mmol} \mathrm{dm}^{-3}$, então nota-se que houve aumento principalmente nas profundidades de 0-0,05 e 0,05-0,10 m, possivelmente em função da adubação e da mineralização de restos culturais. Alleoni, Cambri e Caires (2005), avaliando um Latossolo do cerrado sob plantio direto verificou a mesma tendência após 30 meses de implantação. Embora haja uma preocupação quanto à quantidade de gesso a ser adicionada ao solo, por promover remoção de $\mathrm{K}^{+}$, isso não foi constatado neste trabalho, possivelmente devido ao residual de gesso não proporcionar a remoção de $\mathrm{K}^{+}$.

A acidez ativa do solo, determinada por três métodos, apenas foi influenciada pela profundidade, onde são observadas diferenças nas primeiras três profundidades com médias das duas ultimas profundidades. Considerando que o $\mathrm{pH}$ esta relacionado com a concentração dos íons de $\mathrm{H}^{+}$ na solução do solo, é evidente que ela representa apenas uma pequena relação com a acidez trocável ou acidez potencial do solo. No Brasil em geral considera-se que a faixa ideal de $\mathrm{pH}$ para maioria das culturas está entre 5,7 e 6,0.
Os resultados obtidos nesse trabalho são contrario ao observado por Caires et al. (2002) e Caires et al. (2003), onde esses autores comentam que o gesso após 32 meses proporcionou o aumento do $\mathrm{pH}$ em profundidade abaixo da camada considerada arável. Possivelmente o gesso após 55 meses não apresenta efeito sobre o $\mathrm{pH}$ do solo. $\mathrm{O}$ gesso sendo um sal neutro não interfere diretamente na redução da acidez do solo, apesar de que em alguns casos ocasiona a neutralização de alumínio, isso constitui apenas uma troca de posições de acidez e alcalinidade, que é revertida com o passar do tempo (RAIJ, 2008).

O modelo logístico estima a probabilidade da ocorrência do evento em função das variáveis estudas. Neste estudo o evento em questão é a ocorrência de um nível de alumino que exceda o $\left(0,05 \mathrm{mmol} \mathrm{dm}^{-3}\right)$. Pela figura 1 , a probabilidade de ocorrência de alumínio é superior no plantio convencional quando fixada a profundidade dentro do intervalo 0,05-0,25 $\mathrm{m}$ de profundidade. À medida que se aprofunda no perfil do solo a probabilidade de ocorrência de níveis de alumínio tende aumentar até os $0,25 \mathrm{~m}$. 
Figura 1 Probabilidade de ocorrência de níveis de alumínio no solo em função da profundidade de amostragem para cada sistema de plantio.

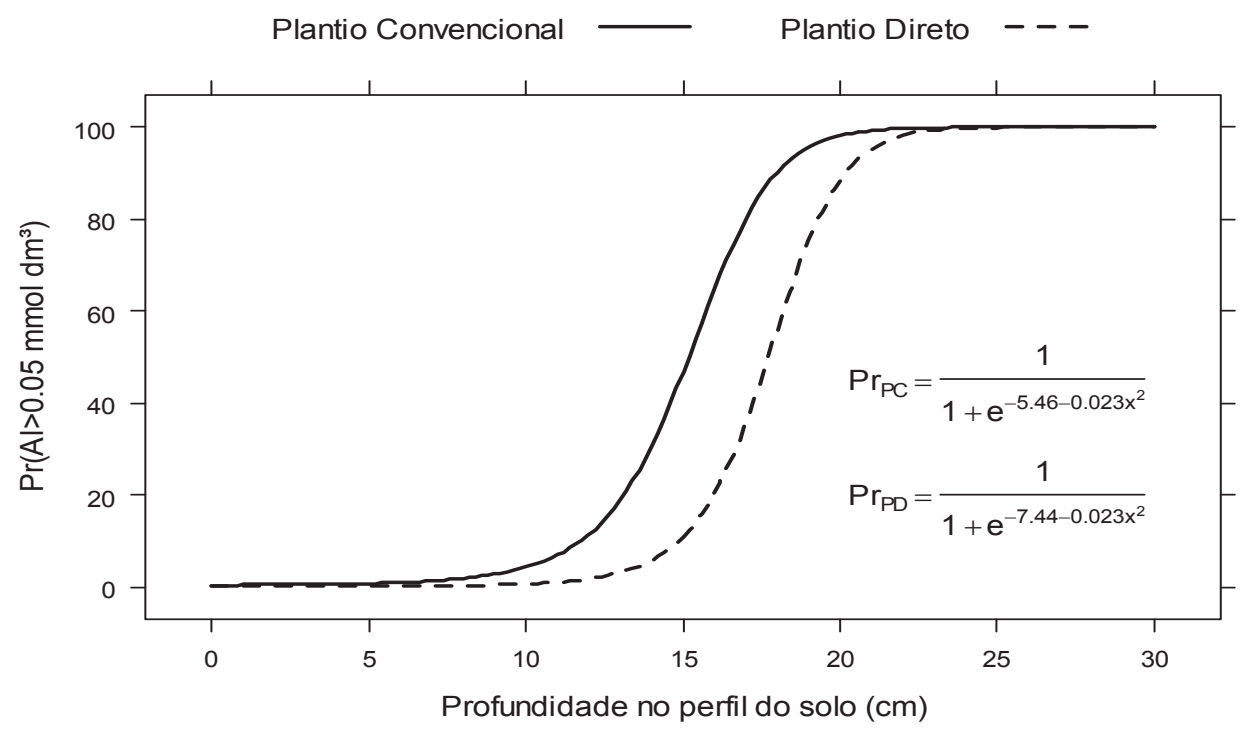

Fonte: Elaboração dos autores.

Observa-se que nas profundidades $0-0,05 \mathrm{~cm}$ não constatou haver probabilidade do teor de $\mathrm{Al}^{3+}$ ser superior ao nível proposto. Enquanto nas outras profundidades, o plantio convencional apresentou maior probabilidade de ocorrência de $\mathrm{Al}^{3+}$. Caires et al. (2008), comentam que a toxicidade causada pelo alumínio em sistemas de plantio direto é reduzida durante os cultivos, e que a toxicidade pelo alumínio pode comprometer o crescimento radicular e a produtividade.

Nos solos cultivados em sistema de plantio direto o efeito tóxico na solução é amenizado pela formação de complexos (quelatos), altamente estáveis com a matéria orgânica. $\mathrm{O}$ aumento de matéria orgânica em plantio direto com passar do tempo, promove incremento dos compostos orgânicos dissolvidos, favorecendo a complexação de alumínio reduzindo a atividade do íon em solução, e consequentemente, sua toxidez às culturas (BAYER; BISSANI; ZANATTA, 2006).

\section{Conclusões}

1- Não houve efeito de gesso para o cálcio, a presença do efeito de gesso aumentou o teor de enxofre e o valor de saturação por base. A acidez ativa do solo e o teor de potássio diminuem conforme se aprofunda no perfil solo.

2- O plantio direto com efeito de gesso aumentou o teor de potássio, enxofre e reduziu a acidez potencial.

3- O plantio convencional com efeito de gesso aumentou a acidez potencial do solo.

4- O plantio direto promoveu aumento de Cálcio, Enxofre e Soma saturação de base.

5- É mais freqüente a obtenção de níveis de alumínio superiores ao nível proposto sob o sistema plantio convencional nas profundidades $0,10-0,15$, $0,15-0,20$ e $0,20-0,30 \mathrm{~m}$. 


\section{Referências}

ALLEONI, L. R. F.; CAMBRI, M. A.; CAIRES, E. F. Atributos químicos de uma Latossolo de cerrado sob plantio direto, de acordo com dose e formas de aplicação de calcário. Revista Brasileira de Ciência do Solo, Viçosa, MG, v. 29, n. 6, p. 923-934, 2005.

ALVA, A. K.; BLAMEY, F. P. C.; EDWARDS, D. G.; ASHER, C. J. An evolution of aluminum indices to predict aluminum toxicity to plants grown in nutrient solutions. Commmuniction Soil Science Plant Analyse, New York, v. 17, n. 12, p. 1271-1280, 1986.

ALVAREZ, V. H. V.; DIAS, L. E.; RIBEIRO JUNIOR, E. S.; SOUZA, R. B.; FONSECA, C. A. Métodos de análise de enxofre em solos e plantas. Viçosa: UFV, 2001. 131 p.

BARTLETT, M. S. Some exemples of statistical methods of research in agriculture and applied biology. Journal of the Royal Statistical Society, London, v. 4, p. 137-183, 1937. Suplemento.

BAYER, C.; BISSANI, C. A.; ZANATTA, J. A. Química de solos em plantio direto. In: FONTOURA, S. M. V.; BAYER, C. Manejo e fertilidade de solos em plantio direto. Guarapuava: Fundação Agrária de Pesquisa Agropecuária, 2006. v. 1, p. 7-29.

BOX, G. E. P.; COX, D. R. The analysis of transformations. Journal of the Royal Statistical Society, London, v. 26, n. 2, p. 211-252, 1964.

BRAGA, J. M.; DEFELIPO, B. V. Determinações espectofométricas de fósforo em extrato de solo e material vegetal. Revista Ceres, Viçosa, v. 113, n. 21, p. 73-85, 1974.

CAIRES, E. F.; CHURKA, S.; GARBUIO, F. J.; FERRARI, R. A.; MORGANO, M. A. Soybean yield and quality as function of lime and gypsum applications. Scientia Agrícola, Piracicaba, v. 63, n. 4, p. 370-379, 2006.

CAIRES, E. F.; KUSMAN, M. T.; BARTH, G.; GARBUIO, F. J.; PADILHA, J. M. Alterações químicas do solo e resposta do milho à calagem e aplicação de gesso. Revista Brasileira de Ciência do Solo, Viçosa, MG, v. 28, n. 1, p. 125-136, 2004.

CAIRES, E. F.; FELDHAUS, I. C.; BARTH, G.; GARBUIO, F. J. Lime and gypsum application on the wheat crop. Scientia Agricola, Piracicaba, v. 59, n. 2, p. 357-364, 2002.

CAIRES, E. F.; GARBUIO, F. J.; CHURKA, S.; BARTH, G.; CORREAA, J. C. L. Effects of soil acidity amelioration by surface liming on no-till corn, soybean, and wheat root growth and yield. European Journal Agronomy, Amsterdam, v. 28, n. 1, p. 57-64, 2008.
CAIRES, E. F.; BANZATTO, D. A.; FONSECA, A. F. Calagem na superfície em sistema plantio direto. Revista Brasileira de Ciência do Solo, Viçosa, MG, v. 24, n. 1, p.161-169, 2000.

CAIRES, E. F.; BLUM, J.; BARTH, G.; GARBUIO, F. J.; KUSMAN, M. T. Alterações químicas do solo e resposta da soja ao calcário e gesso aplicados na implantação do sistema de plantio direto. Revista Brasileira de Ciência do Solo, Viçosa, MG, v. 27, n. 2, p. 275-286, 2003.

CAIRES, E. F.; FELDHAUS, I. C.; BLUM, J. Crescimento radicular e nutrição da cevada em função da calagem e aplicação de gesso. Bragantia, Campinas, v. 60, n. 3, p. 213-223, 2001.

CAIRES, E. F.; FONSECA, A. F.; MENDES, J.; CHUEIRI, W.; MADRUGA, E. F. Produção de milho, trigo e soja em função das alterações das características químicas do solo pela aplicação de calcário e gesso na superfície, em sistema de plantio direto. Revista Brasileira de Ciência do Solo, Viçosa, MG, v. 23, n. 2, p. 315-327, 1999.

CALEGARI, A.; CASTRO FILHO, C.; TAVARES FILHO, J.; RALISCH, R.; GUIMARÃES, M. F. Melhoria da agregação do solo através do sistema plantio direto. Semina: Ciências Agrárias, Londrina, v. 27, n. 2, p. 147-158. 2006.

CAVALIERI, K. M. V.; TORMENA, C. A.; FIDALSKI, J.; PINTRO, J. C.; COSTA, A. C. S.; SOUZA JUNIOR, I. G. Alterações nas propriedades químicas de um Latossolo Vermelho eutroférrico por dois sistemas de manejo de solo. Acta Scientiarum Agronomy, Maringá, v. 26, n. 4, p. 377-385, 2004.

CIOTTA, M. N.; BAYER, C.; FONTOURA, S. M. V.; ERNANI, P. R.; ALBUQUERQUE, J. A. Matéria orgânica e aumento da capacidade de troca de cátions em solo com argila de atividade baixa sob plantio direto. Ciência Rural, Santa Maria, v. 33, n. 6, p. 1161-1164, 2003.

COMPANHIA NACIONAL DE ABASTECIMENTO - CONAB. Brasilian crop assessment: grains: third estimate, december 2008/national supply company. Brasília: Conab, 2008. Disponível em: <http://www. conab.gov.br>. Acesso em: 15 jan. 2009.

COSTA, E. A.; GOEDERT, W. J.; SOUSA, D. M. G. Qualidade de solo submetido a sistemas de cultivo com preparo convencional e plantio direto. Pesquisa Agropecuária Brasileira, Brasília, v. 41, n. 7, p. 11851191, 2006.

COSTA, M. J.; ROSA JUNIOR, E. J.; ROSA, Y. B. C. J.; SOUZA, L. C. F.; ROSA, C. B. J. Atributos químicos e físicos de um Latossolo sendo influenciados pelo 
manejo do solo e efeito da gessagem. Acta Scientiarum Agronomy, Maringá, v. 29, n. 29, p. 701-708, 2007.

DEFELIPO, B. V.; RIBEIRO, A. C. Análise química do solo. Viçosa: UFV, 1981, 17 p. (Boletim de Extensão, 29).

\section{EMPRESA BRASILEIRA DE PESQUISA} AGROPECUÁRIA - EMBRAPA. Centro Nacional de Pesquisa de Solos (Rio de Janeiro, RJ). Manual de métodos de análise de solos. 2. ed. Rio de Janeiro, 1997. $212 \mathrm{p}$.

ERNANI, P. R.; RIBEIRO, M. S.; BAYER, C. Modificações químicas em solos ácidos ocasionadas pelo método de aplicação de corretivos da acidez e de gesso agrícola. Scientia Agricola, Piracicaba, v. 58, n. 4, p. 825831, 2001.

FARINA, M. P. W.; CHANNON, P. Acid-subsoil amelioration. II Gypsum effects on growth subsoil chemical properties. Soil Science Society of America Journal, Madison, v. 52, n. 1, p. 175-180, 1988.

FARINA, M. P. W.; CHANNON, P.; THIBAUD, G. R. Comparison of strategies for ameliorating Subsoil acidity: II. Long-term soil effects. Soil Science Society of America Journal, Madison, v. 64, n. 2, p. 652-658, 2000.

FAVARETTO, N.; NORTON, L. D.; BROUDER, S. M.; JOERN, B. C. Gypsum amendment and exchangeable calcium and magnesium effects on plant nutrition under conditions of intensive nutrient extraction. Soil Science Society of America Journal, Madison, v. 173, n. 2, p. 108118, 2008.

HUSSAIN, I.; OLSON, K. R.; EBELHAR, S. A. Longterm tillage effects on soil chemical properties and organic matter fractions. Soil Science Society of America Journal, Madison, v. 63, n. 5, p. 1335-1341, 1999.

LLANILLO, R. F.; RICHART, A.; TAVARES FILHO, J.; GUIMARÃES, M. F.; FERREIRA, R. R. M. Evolução de propriedades físicas do solo em função dos sistemas de manejo em culturas anuais. Semina: Ciências Agrárias, Londrina, v. 27, n. 2, p. 205-220, 2006.

MARSH, B. H.; GROVE, J. H. Surface and subsurface soil acidity: soybean root response to sulfate-bearing spent lime. Soil Science Society of America Journal, Madison, v. 56, n. 4, p. 1837-1842, 1992.

NARAMABUYE, F. X.; HAYNES, R. J. Effect of organic amendments on soil $\mathrm{pH}$ and $\mathrm{Al}$ solubility and use of laboratory índices to predict their liming effect. Soil Science Society of America Journal, Madison, v. 171, n. 10, p. 754-763, 2006.

NZIGUHEBA, G.; SMOLDERS, E.; MERCKX, R. Sulphur immobilization and availability in soils assessed using isotope dilution. Soil Biology \& Biochemistry, v. 37, n. 4, p. 635-644, 2005.

OATES, K. M.; CALDWELL, A. G. Use of by-product gypsum to alleviate soil acidity. Soil Science Society of America Journal, Madison, v. 49, n. 4, p. 915-918, 1985.

OLIVEIRA, G. C.; DIAS JUNIOR, M. S.; RESCK, D. V. S.; CURI, N. Caracterização química e físico-hídrica de uma Latossolo Vermelho após vinte anos de manejo e cultivo. Revista Brasileira de Ciência do Solo, Viçosa, MG, v. 28, n. 2, p. 327-336, 2004.

PAVAN, M. A.; BINGHAM, F. T.; PRATT, P. F. Redistribution of exchangeable calcium, magnesium, and aluminum following lime and gypsum applications to a Brazilian Oxisol. Soil Science Society of America Journal, Madison, v. 48, n. 1, p. 33-38, 1984.

RAIJ, B. V. Gesso na agricultura. Campionas: Instituto Agronômico de Campinas, 2008. 233 p.

RAIJ, B. V.; QUAGGIO, J. A. Métodos de análise de solos para fins de fertilidade. Campinas: Instituto Agronômico, 1983. 31 p. (Boletim técnico, 81).

SANTOS, J. R.; BICUDO, S. J.; NAKAGAWA, J.; ALBUQUERQUE, A. W. CARDOSO, C. L. Atributos químicos do solo e produtividade do milho afetados por corretivos e manejo do solo. Revista Brasileira de Engenharia Agrícola e Ambiental, Campina Grande, v. 10, n. 2, p. 323-330, 2006.

SATTERTHWAITE, F. E. An approximate distribution of estimates of variance components. Biometrics, Washington, v. 2, n. 6, p. 110-114, 1946.

SCHABENBERGER, O.; PIERCE, F. J. Contemporary statistical models for the plant and soil sciences. Boca Raton, FL: CRC Press, 2002.

SEARLE, S. R.; CASELlA, G.; MUCCLLOCH, C. E. Variance components. New York: John Wiley and Sons, 1992.

SORATTO, R. P.; CRUSCIOL, C. A. C. Atributos químicos do solo decorrente da aplicação em superfície de calcário e gesso em sistema plantio direto recémimplantado. Revista Brasileira de Ciência do Solo, Viçosa, MG, v. 32, n. 2, p. 675-688, 2008.

. Cátions hidrossolúveis na parte aérea de culturas anuais mediante aplicação de calcário e gesso em superfície. Revista Brasileira de Ciência do Solo, Viçosa, MG, v. 31, n. 1, p. 81-90, 2007.

THOMAS, G. A.; DALAL, R. C.; STANDLEY, J. No-till effects on organic matter, $\mathrm{pH}$, cation exchange capacity and nutrient distribution in a Luvisol in the semi-arid subtropics. Soil \& Tillage Research, Netherlands, v. 94, n. 2, p. 295-304, 2007. 
TOMA, M.; SUMMER, M. E.; WEEKS, G.; SAIGUSA, M. Long-term Effects of gypsum on crop yield and subsoil chemical properties. Soil Science Society of America Journal, Madison, v. 39, n. 2, p. 891-895, 1999.

TRIPLETT, G. B. J.; DICK, W. A. No-Tillage crop production: a revolution in agriculture. Agronomy Journal, Madison, v. 100, p. 153-165, 2008. Supplement 3.

VETTORI, L. Métodos de análise de solos. Rio de Janeiro: Equipe de pedologia e fertilidade do solo do
Ministério da Agricultura, 1969. 24 p. (Boletim técnico, 7).

VITTI, C. G.; LUZ, P. H. C.; MALAVOLTA, E.; DIAS, A. S.; SERRANO, C. G. E. Uso do gesso em sistemas de produção agrícola. Piracicaba: GAPE, 2008. 104 p.

ZAMBROSI, F. C. B.; ALLEONI, L. R. F.; CAIRES, E. F. Teores de alumínio trocável e não trocável após calagem e gessagem em Latossolo sob sistema plantio direto. Bragantia, Campinas, v. 66, n. 3, p. 487-495, 2007. 
